\title{
SUMS OF DISTANCES BETWEEN POINTS OF A SPHERE
}

\author{
GLYN HARMAN \\ Department of Mathematics, Royal Holloway College \\ Egham, Surrey TW20 OEX, England \\ (Received February 1, 1982)
}

ABSTRACT. Given $\mathrm{N}$ points on a unit sphere in $\mathrm{k}+\mathrm{l}$ dimensional Euclidean space, we obtain an upper bound for the sum of all the distances they determine which improves upon earlier work by K. B. Stolarsky when $k$ is even. We use his method, but derive a variant of W. M. Schmidt's results for the discrepancy of spherical caps which is more suited to the present application.

KEY WORDS AND PHRASES. Geometrical inequalities, extremum problems, irregularities of distribution.

1980 MATHEMATICS SUBJECT CLASSIFICATION CODES. 52A40; $10 K 30$.

\section{INTRODUCTION.}

In this paper we shall consider the following interesting problem of a geometrical nature: Given a function which measures the distance between points on the unit sphere in $\mathrm{m}$-dimensional space, for what set of $\mathrm{N}$ points on the sphere is the sum of all distances between points a maximum, and what is the maximum? K. B. Stolarsky made important progress towards the solution of this problem in [3]. We refer the reader to his paper for the history of earlier work. He showed (see Theorem 2 below), for a large class of distance measuring functions, that the sum of the distances between points plus a measure of how far the set of points deviates from uniform distribution is equal to $N^{2} c(f, m)$. Here $c$ is a constant depending 
only on the function $f$ used to measure the distances, and the dimension $m$ of the space. The sum of distances is thus maximised by a well distributed set of points. By examining carefully the estimation of the discrepancy of the point distribution we shall obtain results which, in certain cases, are very near to being best possible. The best possible result in this context is that the maximum sum of distances between $\mathrm{N}$ points is

$$
c(f, m) N^{2}-h(f, m) N^{1-1 /(m-1)}
$$

We now introduce some notation following [3] to make the above statements more precise. Write $U$ for the surface of the unit sphere in $k+l$ dimensional Euclidean space $E^{k+l}$. Let $M_{p}$ be a sequence of $N$ points $p_{1}, \ldots, p_{N} \varepsilon U$. For a function $d$ on $U \times U$ we define

$$
s\left(N, k, M_{p}\right)=s\left(d ; N, k, M_{p}\right)=\sum_{i<j} d\left(p_{i}, p_{j}\right)
$$

and

$$
S(N, k)=S(d ; N, k)=\max S\left(d ; N, k, M_{p}\right)
$$

where the maximum in (2) is taken over all sequences $M_{p} \cdot K$. B. Stolarsky has shown (see Theorem 2 below) that the sum in (1) plus an integral which measures the discrepancy of $M_{p}$ equals a constant depending only on $d$, when $d$ belongs to a certain class of functions. This class includes the usual Euclidean distance function.

We write $p_{0}$ for the vector $(1,0, \ldots 0)$ in $E^{k+1}$. For a function $f$ of a vector $P \varepsilon U$ we write

$$
\int(\tau p) d \tau
$$

for the integral of $f$ over the special orthogonal group acting on $U$. Here $\tau$ represents an orthogonal transformation. For a positive function $g$ of a real variable $x \in[0,1]$ we define a distance function $\rho$ (which can be shown to be a metric) by 


$$
\rho\left(\mathrm{p}_{1}, \mathrm{p}_{2}\right)=\rho\left(\mathrm{p}_{1}, \mathrm{p}_{2} ; \mathrm{g}\right)=\int\left(\int_{\tau \mathrm{p}_{1} \cdot \mathrm{p}_{\mathrm{O}}}^{\tau \mathrm{p}_{2} \cdot \mathrm{P}_{\circ}} \mathrm{g}(\mathrm{x}) \mathrm{dx}\right) \mathrm{d \tau}
$$

for points $\mathrm{P}_{1}, \mathrm{P}_{2} \varepsilon \mathrm{U}$, whenever the integral in ( 3 ) exists (i.e. when the inner integral is intergrable over the orthogonal group). Here p.q denotes the standard inner product of two vectors. We call $g$ the kernel of $\rho$.

Let $\sigma(U)$ denote the surface area of $U$. Write $d_{0}\left(q_{1}, q_{2}\right)$ for the great circle metric and $d_{1}\left(q_{1}, q_{2}\right)$ for the great circle metric defined by (3) with kernel $\left(1-x^{2}\right)^{-\frac{1}{2}}$. It is shown in [3] that

$$
d_{1}\left(q_{1}, q_{2}\right) \leqq d_{0}\left(q_{1}, q_{2}\right)
$$

Henceforth $d\left(q_{1}, q_{2}\right)$ will be the usual Euclidean metric. Our main result is as follows :

THEOREM 1. For $k$ even we have

$$
c_{1}(k) N^{2}-c_{2}(k) N^{1-1 / k}<s\left(d_{1} ; N ; k\right)<c_{1}(k) N^{2}-c_{3}(k) N^{1-1 / k}(\log N)^{-1} .
$$

For $k$ even and $\varepsilon>0$ we have

$$
C_{4}(k) N^{2}-C_{5}(k) N^{1-1 / k}<S(d ; N, k)<C_{4}(k) N^{2}-C_{6}(k, \varepsilon) N^{1-2 / k}(\log N)^{-1-\varepsilon-1 /(k+l)} .
$$

For the right hand sides of (4) and (5) Stolarsky gives

$$
C_{1}(k) N^{2}-C_{3}(k, \varepsilon) N^{1-2 / k-1 / k^{2}-\varepsilon} \quad \text { and } \quad C_{4}(k) N^{2}-C_{6}(k, \varepsilon) N^{1-3 / k-2 / k^{2}-\varepsilon}
$$

respectively. For $k$ odd the only improvement we can offer is the replacement of $\mathrm{N}^{\varepsilon}$ by a power of $\log \mathrm{N}$.

The main result of Stolarsky's paper which we use is

THEOREM 2 (Stolarsky). We have

$$
S\left(\rho ; N, k, M_{p}\right)+\int_{-1}^{l} g(x) \int\left(f\left(M_{p}, \tau, x\right)-N \sigma^{*}(x)\right)^{2} d \tau d x=\frac{N^{2}}{2 \sigma(U)^{2}} \iint \rho(p, q) d \sigma(q) .
$$

Here $f\left(M_{p}, \tau, x\right)$ is the number of points of $M_{p}$ in the spherical cap: 
$\left\{\mathrm{p} \varepsilon \mathrm{U}: \mathrm{p} \cdot \tau \mathrm{p}_{\mathrm{O}} \leq \mathrm{x}\right\}$. Also $\sigma^{*}(\mathrm{x})$ denotes the normalized surface area of a spherical cap, radius $x$, and $d \sigma(q)$ represents an element of surface area on $U$. The second term on the left of $(6)$ is clearly a measure of the discrepancy of $M_{p}$. In the second section we shall show how to obtain an estimate for a related measure, and in our section 3 we shall prove Theorem 1 .

\section{SCHMIDT'S INTEGRAL EQUATION METHOD}

In the following, constants implied by the $\ll$ notation shall depend only on k. We will make two changes to Schmidt's method. At one stage (p.69 of [1]) for $k$ even he uses the inequality $1+\log (1 / r) \ll r^{-1}$ which suffices to prove his results, but which is wasteful in our present context. This produces the improvement in the exponent of $\mathrm{N}$ over Stolarsky's results. To improve $\mathrm{N}^{\varepsilon}$ to a power of $\log \mathrm{N}$ we do not allow constants introduced to depend on a parameter $\alpha$. This enables us to choose $\alpha$ as a function of $N$, whereas Schmidt could only choose $\alpha$ as a function of $k$ and $\varepsilon$.

As in [1] and [3] (section 4) we let $\mu$ be the normalized Lebesgue measure on $U($ so $\mu(u)=1)$. We write $C(r, p)$ for the spherical cap of all points on $U$ whose spherical distance from $p \varepsilon U$ is no more than $r$. Put $\nu(C(r, p))$ for the number of points of $M_{p}$ in $C(r, p)$ and $D(r, p)=N \mu(C(r, p))-\nu(C(r, p))$.

We write

$$
E(r, s)=\int_{U} D(r, p) D(s, p) d \mu(p)
$$

We note that

$$
E(r, r) \ll N^{2} r^{k}
$$

The main result of this section $i$

THEOREM 3. We have, for $N \delta^{k} \geqq ?, k$ even, that

$$
\int_{0}^{\delta}\left(\log \left(N \delta^{k}\right)+\log (\delta / r)\right) E(r, r) d r>N^{1-l / k} \delta^{k} .
$$

We note here that all the results in [1] may be improved by the replacing of $\left(N \delta^{k}\right)^{\varepsilon}$ with $\log \left(\mathrm{N} \delta^{\mathrm{k}}\right)$, or $\left(\log \left(\mathrm{N} \delta^{\mathrm{k}}\right)\right)^{\frac{1}{2}}$. That these results are very ncar to being best 
possible is shown in [3].

The idea of Schmidt's method as we use it here is to show that for $0<\alpha<1$, $\delta^{-1} \int_{0}^{\delta}\left((1-\alpha)^{-1}+\log (\delta / r)\right) E(r, r) d r>\int_{0}^{c^{\prime}} r^{1-k-\alpha} E(\delta r, \delta r) d r$

where $c^{\prime}$ is a suitable small constant. The use of the apparently trivial inequality

$$
E(\delta r, \delta r)>>\|N \mu(C(p, \delta r))\|^{2}
$$

then gives a lower bound

$$
(I-\alpha) \delta^{k+\alpha-2} N^{l+\alpha / k-2 / k}
$$

for the right hand side of (9) (see p.82 of [1]). The choice of $\alpha$ as $1-\left(\log N \delta^{k}\right)^{-1}$ then gives (8).

We now outline how to obtain (9), referring to [1] (the reader may also follow the argument using [2]). Put $\beta=1-\alpha$ and write

$$
J=\delta^{-I} \int_{r+s \leqq I}^{\delta} \int_{s}^{\delta} E(r, s) \cos \frac{r-s}{2} \cos \frac{r+s}{2}\left(\sin \left|\frac{r-s}{2}\right|\right)^{-\alpha}\left(\sin \frac{r+s}{2}\right)^{-\beta} d r d s .
$$

Then (p.78 of $[1]$ we have

$$
\begin{aligned}
J & \ll \int_{\substack{r+s \leq 1 \\
r+1}}^{1} E(\delta r, \delta r)|r-s|^{-\alpha}|r+s|^{-\beta} d r d s \\
& =\int_{0}^{1} d r E(\delta r, \delta r) \int_{0}^{1 / r-1} d t|1-t|^{-\alpha}|1+t|^{-\beta} \\
& <\int_{0}^{1}\left((1-\alpha)^{-1}+\log (1 / r)\right) E(\delta r, \delta r) d r \\
& =\delta^{-1} \int_{0}^{\delta}\left((1-\alpha)^{-1}+\log (\delta / r)\right) E(r, r) d r .
\end{aligned}
$$

Now, by Lemma 6 of [1], 


$$
J=\int_{0}^{\frac{1}{2}} E(\delta r, \delta r) f(r) d r
$$

where $f$ satisfies a certain integral equation. It is shown in [1], pages 79-81 that

$$
f=f_{0}(r)-f_{*}(r) .
$$

Here, in Schmidt's notation

$$
f_{0}(r)><r^{1-\alpha}, f_{*}(r)><r^{1-k-\alpha}
$$

as $\mathrm{r} \rightarrow 0$, uniformly in $\delta$. The tracing of the dependence of all the constants in [1] on $\alpha$ is tedious but straightforward. They are all bounded above and below by positive constants independent of $\alpha$ (this is not true for $k$ odd, but in that case we get $f_{0}(r)>\left\langle(1-\alpha)^{-1} r^{-\alpha}\right.$ which is good enough to prove the corresponding result). We see that (9) follows from (11), (12) and (13), and the proof of the theorem is complete.

\section{PROOF OF THEOREM 1}

The lower bounds in (4) and (5) are established in [3]; we have included them here for completeness.

(i) Proof of (4). We have

$$
\begin{aligned}
& \int_{-1}^{1} g(x) \int\left(f\left(M_{p}, \tau, x\right)-N \sigma^{*}(x)\right)^{2} d \tau d x \\
& >>\int_{-1}^{1}\left(1-x^{2}\right)^{-\frac{1}{2}} E(\arccos |x|, \arccos |x|) d x \\
& >>\int_{0}^{\pi / 2} E(r, r) d r .
\end{aligned}
$$

Now, by (8) 


$$
\int_{0}^{\pi / 2}(\log N+\log (\pi / 2 r)) E(r, r) d r>>N^{1-1 / k}
$$

Put $n=N^{-1}$. Then, by (7),

$$
\int_{0}^{n}(\log N+\log (\pi / 2 r)) E(r, r) d r \ll N^{2}((\log N)+|\log n|) n^{k+1}=o(1) .
$$

So, by (15),

$$
\begin{aligned}
\int_{0}^{\pi / 2} E(r, r) d r & \gg(\log N)^{-1} \int_{n}^{\pi / 2}(\log N+\log (\pi / 2 r)) E(r, r) d r \\
& \gg N^{1-1 / k}(\log N)^{-1} .
\end{aligned}
$$

With (14) and (6) this proves Theorem 1 for this case.

(ii) Proof of (5). In this case we have (see 4.7 of [3]),

$$
\int_{-1}^{l} g(x) \int\left(f\left(M_{P}, \tau, x\right)-N \sigma^{*}(x)\right)^{2} d \tau d x>\eta \int_{\eta}^{\pi / 2} E(r, r) d r .
$$

This time we choose $\eta$ by

$$
\eta=N^{-1 / k}(\log N)^{-1 /(k+1)-\varepsilon} \text {. }
$$

Combining (15), (16) and (6) completes the proof of (5) since

$$
\int_{0}^{n}(\log N+\log (\pi / 2 r)) E(r, r) d r=o\left(N^{1-1 / k}\right) .
$$

ACKNOWLEDGEMENT. I should like to take this opportunity to thank R. C. Baker for suggesting this problem to me, and K. B. Stolarsky for encouraging me to write this paper. This research was carried out while I held a London University Postgraduate Studentship.

\section{REFERENCES}

1. SCHMIDT, W. M. "Irregularities of Distribution IV" Inventiones Math. 7, 55-82, 1969. 
2. SCHMIDT, W. M. Lectures on irregularities of distribution (Tata Institute, Bombay, 1977).

3. STOLARSKY, K. B. "Sums of distances between points on a sphere II" Proc. Am. Math. Soc. 41, 575-582, 1973. 


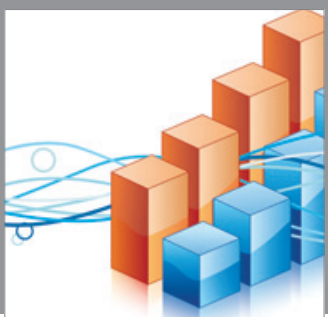

Advances in

Operations Research

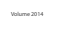

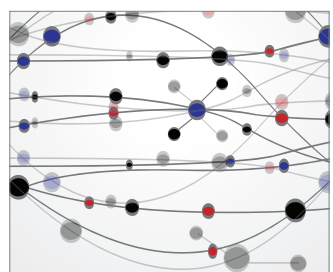

\section{The Scientific} World Journal
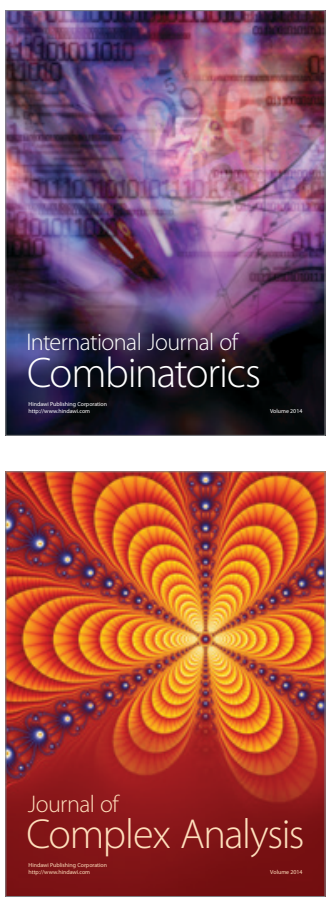

International Journal of

Mathematics and

Mathematical

Sciences
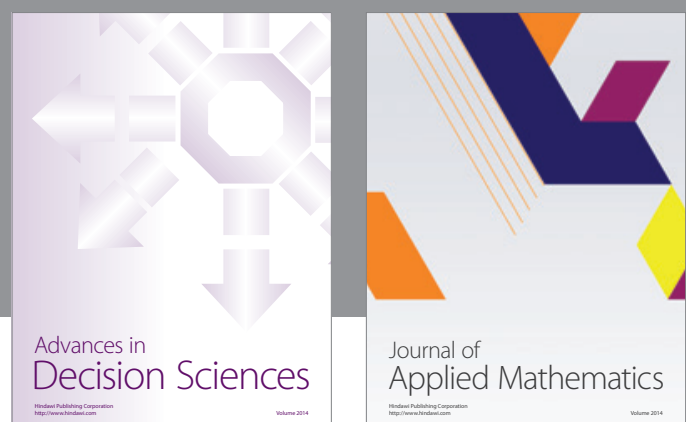

Journal of

Applied Mathematics
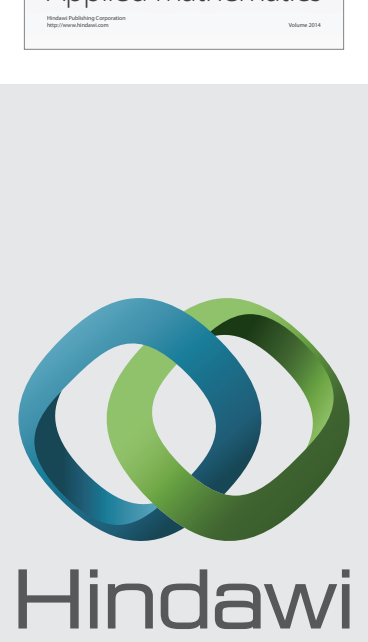

Submit your manuscripts at http://www.hindawi.com
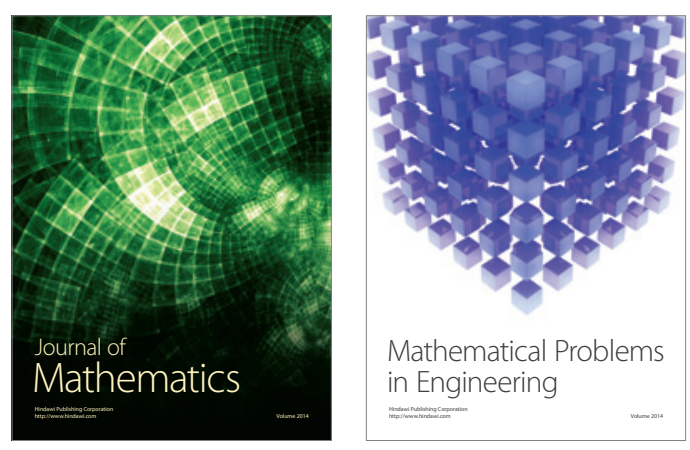

Mathematical Problems in Engineering
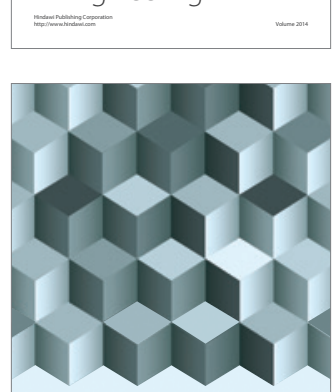

Journal of

Function Spaces
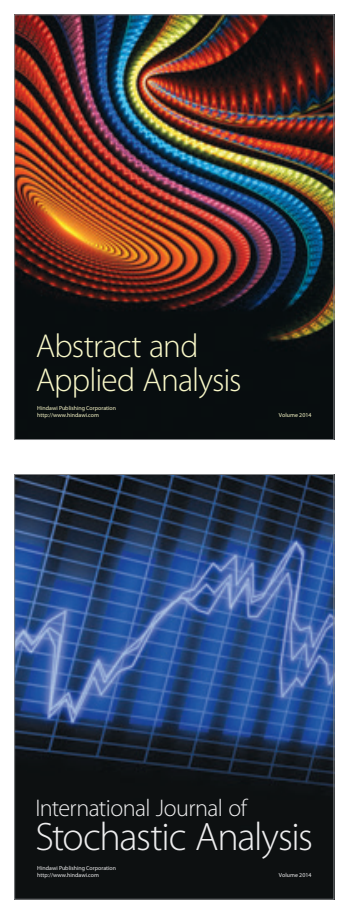

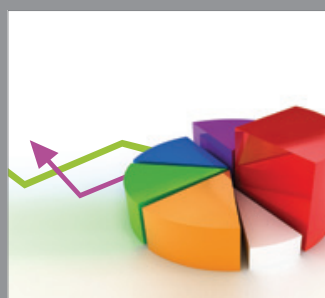

ournal of

Probability and Statistics

Promensencen
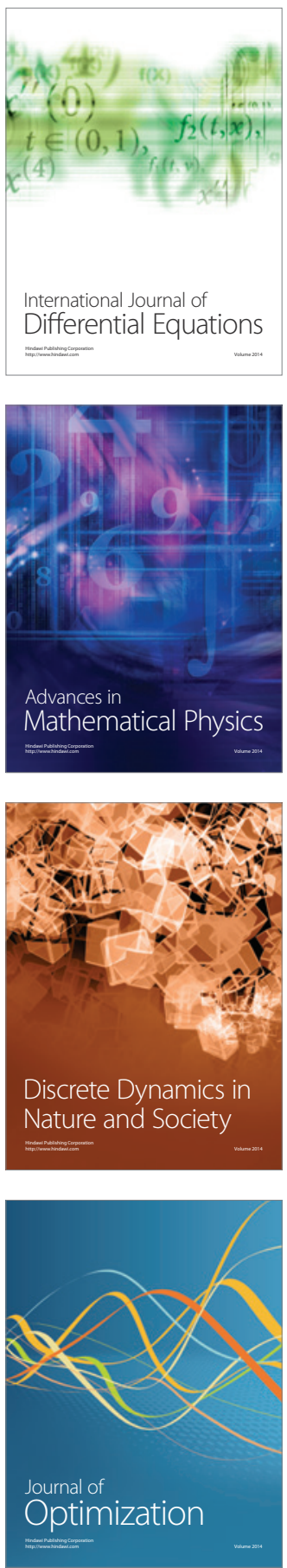